CRYSTALLOGRAPHIC COMMUNICATIONS

ISSN 2056-9890

Received 26 March 2018

Accepted 29 March 2018

Edited by J. Simpson, University of Otago, New Zealand

Keywords: crystal structure; ethenes; ferrocene.

CCDC reference: 1833400

Supporting information: this article has supporting information at journals.iucr.org/e

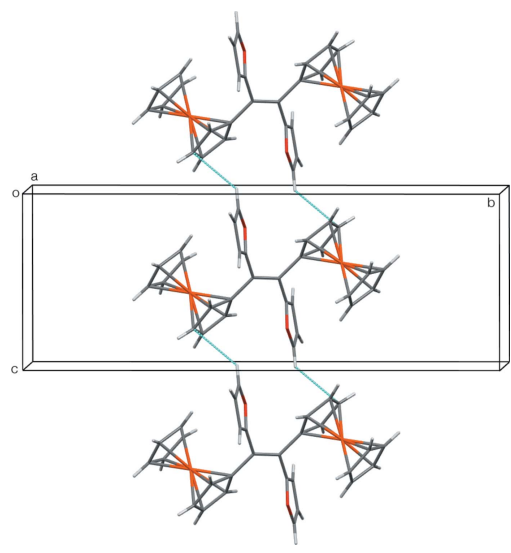

OPEN $\odot$ ACCESS

\section{Crystal structure of $(E)$-1,2-diferrocenyl-1,2- bis(furan-2-yl)ethene}

\author{
Anthony Linden, ${ }^{\mathrm{a} *}$ Róża Hamera-Fałdyga, ${ }^{\mathrm{b}}$ Grzegorz Mlostoń ${ }^{\mathrm{b}}$ and Heinz \\ Heimgartner ${ }^{a}$
}

\footnotetext{
${ }^{\mathbf{a}}$ Department of Chemistry, University of Zurich, Winterthurerstrasse 190, CH-8057 Zurich, Switzerland, and bepartment of Organic and Applied Chemistry, University of Łódź, Tamka 12, PL-91-403 Łódź, Poland.

*Correspondence e-mail: anthony.linden@chem.uzh.ch
}

The title compound, $\left[\mathrm{Fe}_{2}\left(\mathrm{C}_{5} \mathrm{H}_{5}\right)_{2}\left(\mathrm{C}_{20} \mathrm{H}_{14} \mathrm{O}_{2}\right)\right]$, is the product of a new synthetic route towards tetraaryl/hetaryl-substituted ethenes that reduces the occurrence of side-products. In the crystal, the molecule is centrosymmetric and the cyclopentadienyl (Cp) rings are nearly coplanar and aligned slightly closer to a staggered conformation than to an eclipsed one. The ethene plane is tilted by $32.40(18)^{\circ}$ with respect to that of the substituted Cp ring and by $63.19(19)^{\circ}$ with respect to that of the furan ring. $\mathrm{C}-\mathrm{H} \cdots \pi$ interactions link the molecules into a three-dimensional supramolecular framework.

\section{Chemical context}

Tetrasubstituted ethenes bearing aryl, hetaryl or ferrocenyl groups are of current interest, as many of them find applications as novel materials for photooptics, electronics, crystal engineering and as new medications (Astruc, 2017). Ethene derivatives with a ferrocenyl unit on one or both $\mathrm{C}$ atoms of the alkene deserve special attention. Prominent representatives of the first type are ferrocifene $\{1$-[4-(2-dimethylaminoethoxy)phenyl]-1-phenyl-2-ferrocenylbut-1-ene $\}$ and its di$\mathrm{OH}$ analogue, which are known as potent, organometallic antitumor drugs (Jaouen et al., 2015; Resnier et al., 2017). On the other hand, dimethyl $(Z)$-2,3-diferrocenylbut-2-enedioate displays interesting redox and solvatochromic properties (Solntsev et al., 2011). As typical procedures for the preparation of tetrasubstituted ethenes containing a ferrocenyl substituent, conversions of the corresponding ketones under the McMurry reaction conditions (Top et al., 1997) or reductive coupling using low-valent titanium agents are recommended (Dang et al., 1990). In both cases, the reported yields are satisfactory to good, but a serious disadvantage is the formation of side-products. Recently, we reported a new approach to tetraaryl/hetaryl-substituted ethenes via desilylation of 2-(trimethylsilyl)-4,4,5,5-tetraaryl/hetaryl-1,3-dithiolanes, obtained from diaryl/hetaryl thioketones by treatment with (trimethylsilyl)diazomethane $\left(\mathrm{TMS}-\mathrm{CHN}_{2}\right)$ at low temperature (Mloston et al., 2017). The mechanism of this unusual conversion was explained by the assumption that the in situ-generated 1,3-dithiolane anion undergoes a spontaneous cycloelimination $([3+2]$-cycloreversion $)$ to give the dithioformate anion and the corresponding tetrasubstituted ethene derivative. The same method was applied for the preparation of some ferrocenyl/hetaryl-substituted ethenes (Mlostoń et al., 2018). 
Here we report the analogous synthesis and crystal structure of the known title compound, $(E)-1$, with m.p. 485-487 K. For the previously described synthesis of this product (Dang et al., 1990), a m.p. of $489-491 \mathrm{~K}$ and a yield of $17 \%$ were reported and the authors tentatively assigned the $(E)$-configuration to the obtained compound. In our case, single crystals of $(E)-\mathbf{1}$ were grown from hexane $/ \mathrm{CH}_{2} \mathrm{Cl}_{2}$ and used for an $\mathrm{X}$-ray diffraction analysis, from which the previous tentatively postulated structure of the obtained isomer could be confirmed.

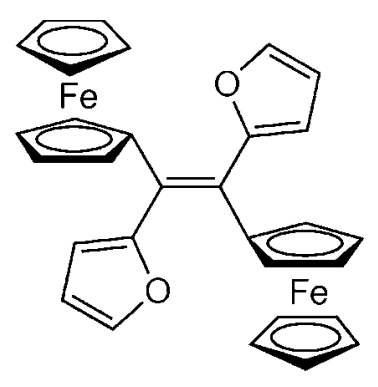

\section{Structural commentary}

The molecule of $(E)-\mathbf{1}$ sits across a crystallographic centre of inversion and is shown in Fig. 1. Within the asymmetric unit, the $\mathrm{Fe}$ atom sits very well centred between the cyclopentadienyl $(\mathrm{Cp})$ rings with all $\mathrm{Fe}-\mathrm{C}$ distances in the range 2.0352 (17)-2.0712 (16) А. The Cp C-C bond lengths [mean 1.435 (2) A] involving the substituted $\mathrm{C}$ atom, C6, are very slightly elongated compared with the other $\mathrm{C}-\mathrm{C}$ distances [mean 1.418 (3) $\AA$ ] . Other bond lengths and angles are unremarkable. The two $\mathrm{Cp}$ rings are aligned slightly closer to a staggered conformation than to an eclipsed one, with the ring rotation from perfectly eclipsed being $20.6(2)^{\circ}\left(18^{\circ}\right.$ is the halfway point between eclipsed and staggered). The dihedral

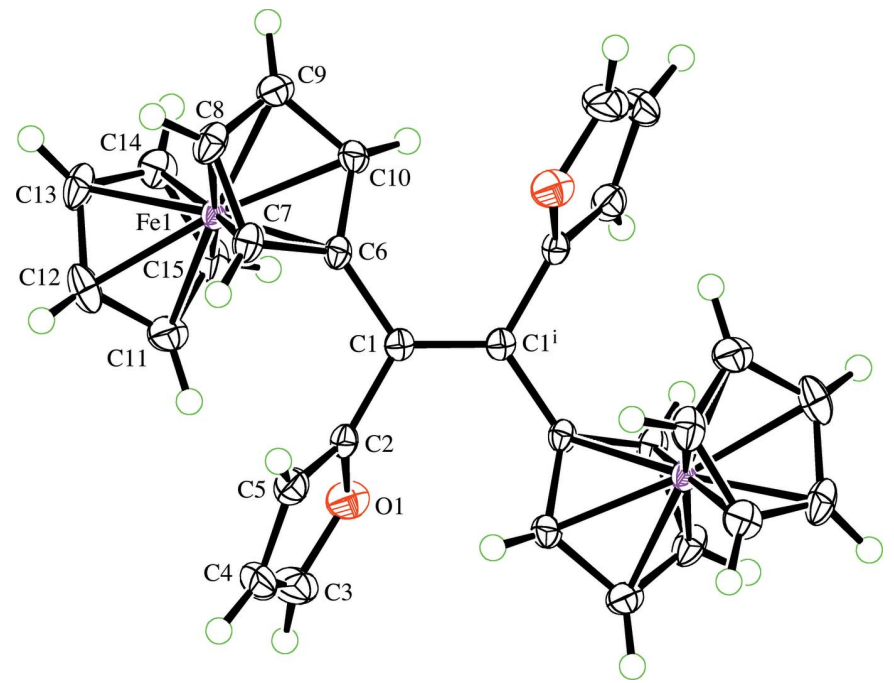

Figure 1

The molecular structure of the title compound, $(E)-\mathbf{1}$, showing the atomnumbering scheme. Displacement ellipsoids are drawn at the $50 \%$ probability level. Symmetry code: (i) $-x+1,-y+1,-z+1$.
Table 1

Hydrogen-bond geometry $\left(\AA,^{\circ}\right)$.

$C g 1, C g 2$ and $C g 3$ are the centroids of the $\mathrm{C} 6-\mathrm{C} 10, \mathrm{C} 11-\mathrm{C} 15$ and $\mathrm{C} 2 / \mathrm{O} 1 / \mathrm{C} 3-$ C5 rings, respectively.

\begin{tabular}{lllll}
\hline$D-\mathrm{H} \cdots A$ & $D-\mathrm{H}$ & $\mathrm{H} \cdots A$ & $D \cdots A$ & $D-\mathrm{H} \cdots A$ \\
\hline $\mathrm{C} 3-\mathrm{H} 3 \cdots C g 1^{\mathrm{i}}$ & 0.95 & 2.81 & $3.686(3)$ & 153 \\
$\mathrm{C} 8-\mathrm{H} 8 \cdots C g 2^{\mathrm{ii}}$ & 0.95 & 2.85 & $3.764(2)$ & 161 \\
$\mathrm{C} 10-\mathrm{H} 10 \cdots C g 3^{\mathrm{iii}}$ & 0.95 & 2.68 & $3.2097(18)$ & 116 \\
\hline
\end{tabular}

Symmetry codes: (i) $x, y, z+1$; (ii) $x+\frac{1}{2},-y+\frac{3}{2}, z-\frac{1}{2}$; (iii) $-x+1,-y+1,-z+1$.

angle between the planes of the two $\mathrm{Cp}$ rings in the ferrocenyl entity is only $4.08(11)^{\circ}$ and ethene atom $\mathrm{C} 1$ is coplanar with the $\mathrm{Cp}$ ring to which it is bonded. However, the ferrocenyl entity is tilted with respect to the ethene plane, with a dihedral angle between the plane of the substituted $\mathrm{Cp}$ ring and that of the ethene plane of $32.40(18)^{\circ}$. The dihedral angle between the substituted $\mathrm{Cp}$ ring and the adjacent furan ring is $53.46(11)^{\circ}$, while that between the plane of the furan ring and the ethene plane is $63.19(19)^{\circ}$. The planes of the two furan rings are necessarily parallel because of the centre of inversion.

\section{Supramolecular features}

There are no significant $\mathrm{C}-\mathrm{H} \cdots \mathrm{O}$ or $\pi-\pi$ interactions, but some weak $\mathrm{C}-\mathrm{H} \cdots \pi$ interactions are present (Table 1). C8$\mathrm{H}$ of the substituted $\mathrm{Cp}$ ring has an edge-on intermolecular interaction with the unsubstituted $\mathrm{Cp}$ ring at $x+\frac{1}{2},-y+\frac{3}{2}, z-\frac{1}{2}$. The extension of this interaction through the molecular centre of inversion leads to sheets of molecules, which lie parallel to the (101) plane (Fig. 2). The furan ring, via $\mathrm{C} 3-\mathrm{H}$, has an edge-on intermolecular $\mathrm{C}-\mathrm{H} \cdots \pi$ interaction with the substituted $\mathrm{Cp}$ ring at $x, y, z+1$. This interaction leads to double-stranded chains or ladders, in which the molecule acts as the ladder rungs; the chains run parallel to the [001]

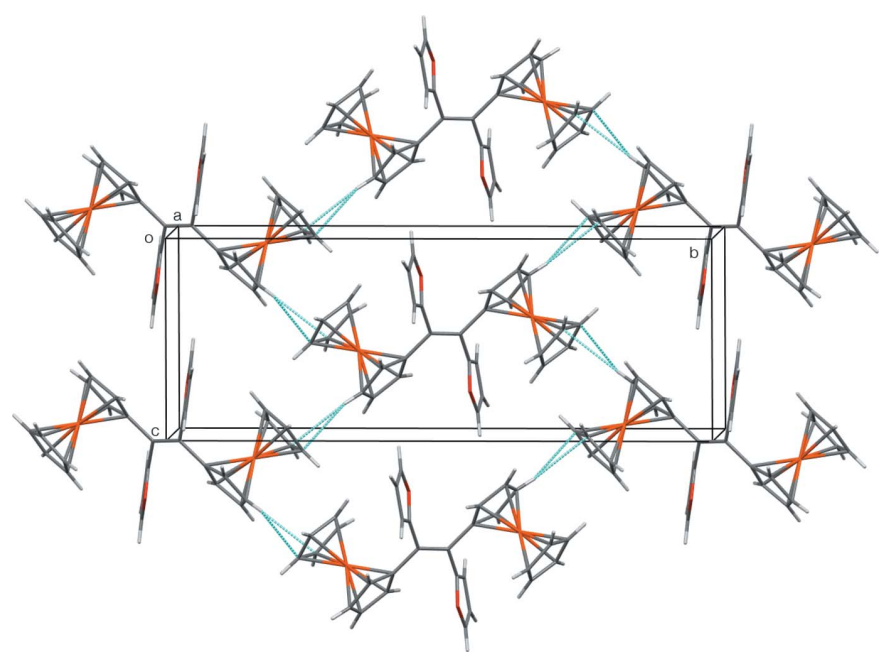

Figure 2

The sheets of molecules lying parallel to the (101) plane formed by the $\mathrm{C} 8-\mathrm{H} \cdots \pi$ interactions. 


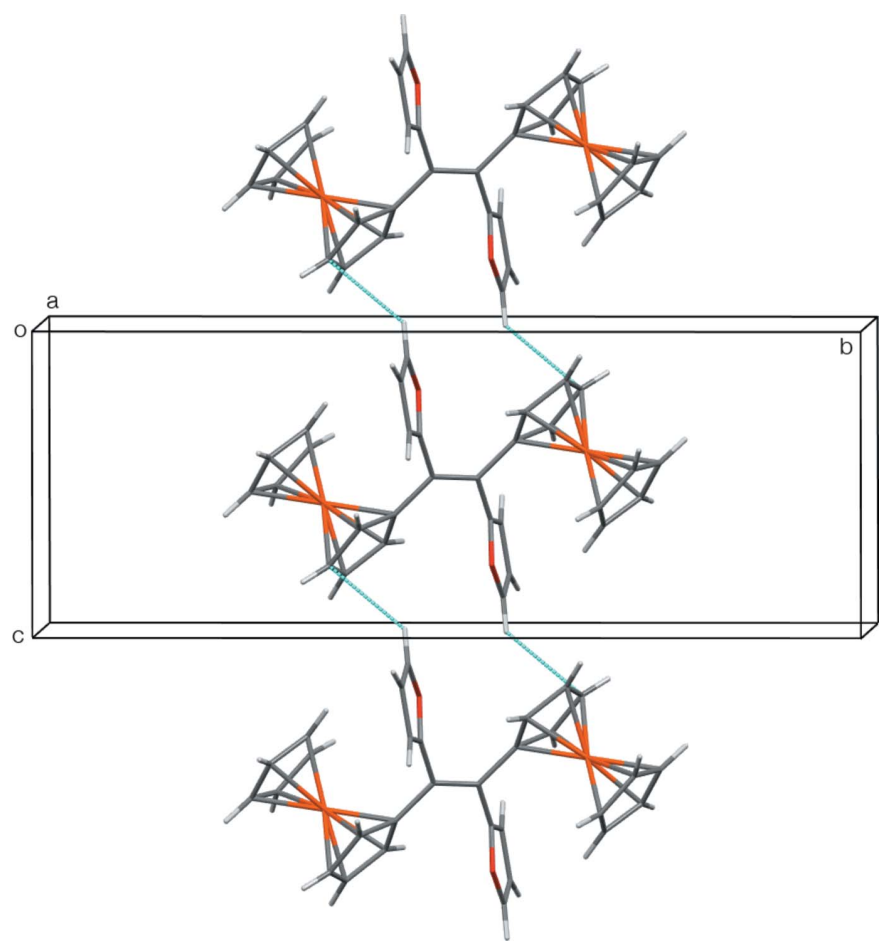

Figure 3

The ladder motif running parallel to [001] formed by the $\mathrm{C} 3-\mathrm{H} \cdots \pi$ interactions.

direction (Fig. 3). Finally, $\mathrm{C} 10-\mathrm{H}$ of the substituted $\mathrm{Cp}$ ring interacts intramolecularly with the $\pi$-system of the furan ring at $-x+1,-y+1,-z+1$ on the opposite side of the molecule. This latter interaction is quite short, but has a sharp angle at the $\mathrm{H}$ atom (Table 1), so the arrangement might just be a consequence of the molecular conformation. The molecular inversion symmetry, in combination with the two types of intermolecular interactions, links the molecules into a threedimensional supramolecular framework.

\section{Database survey}

The Cambridge Structural Database (CSD, Version 5.39 with February 2018 updates; Groom et al., 2016) contains one entry for a 1,1-diferrocenylethene [1,1-bis $\left(1^{\prime \prime}, 2^{\prime \prime}, 3^{\prime \prime}, 4^{\prime \prime}, 5^{\prime \prime}\right.$-pentamethylferrocen-1'-yl)ethene, CSD refcode CIJQAN, Heigl et al., 1999] and 24 entries involving related 1,2-diferrocenylethenes, 10 of which are $(E)$-isomers. The archetypal structure is (E)-1,2-diferrocenylethene (REBDAD, Denifl et al., 1996), in which the $\mathrm{Cp}$ rings of the ferrocenyl entities adopt an almost perfectly eclipsed arrangement. With the exception of 1-(1'benzoylferrocenyl)-2-ferrocenylethene and 1-(1'-(4-methoxybenzoyl)ferrocenyl)-2-ferrocenylethene (OJUWUN and OJUXAU, Roemer et al., 2016), in which the ferrocenyl Cp rings lie close to a staggered arrangement, all of the other structures of molecules with the $(E)$-configuration display $\mathrm{Cp}$ arrangements that are much closer to eclipsed than observed for (E)-1 (ACUVAV, Mata \& Peris, 2001; IBAXAM, DeHope et al., 2011; IVOSER, Skibar et al., 2004; JANJAJ, Dong et al., 1989; OJUXEY, Roemer et al., 2016; QICKIW, Chen et al.,
2000; REBDAD; WIMYOH, Nagahora et al., 2007, Roemer \& Lentz, 2008, Farrugia et al., 2009). The 1,1-diferrocenylethene structure also has eclipsed $\mathrm{Cp}$ rings. The two staggered $(E)$ configured examples have a bulky substituent on one of the distal Cp rings; those with a less bulky $\mathrm{Cp}$ substituent have the eclipsed arrangement. Interestingly, $(E)-\mathbf{1}$ has no Cp substituents yet the $\mathrm{Cp}$ ring arrangement deviates significantly from eclipsed. The degree of eclipsing of the $\mathrm{Cp}$ conformations found among the molecules with the $(Z)$-configuration, two of which have a cyclopropene ring as the ethene bridge (AMODIP, Klimova, Berestneva, Ramirez et al., 2003; EQOMIG, Klimova, Berestneva, Cinquantini et al., 2003), is more varied (AMODOV and AMODUB, Klimova, Berestneva, Ramirez et al., 2003; BADDAM, Beletskaya et al., 2001, Solntsev et al., 2011; JAJYIF and JAJYOL, García, FloresAlamo, Flores \& Klimova, 2017; KIGQUO, Klimova et al., 2013; LUFCEW, García et al., 2014; QASPEI, QATDAT and QATDEX, García, Flores-Alamo, Ortiz-Frade \& Klimova, 2017; QICKOC, Chen et al., 2000; TUJDEI, Klimova et al., 2009).

\section{Synthesis and crystallization}

The title compound was prepared according to the reaction sequence presented in the scheme below. A solution of thioketone 2 (297 mg, $1 \mathrm{mmol}$; prepared according to Mlostoń et al., 2015) in THF (3 ml) was cooled to $198 \mathrm{~K}$ (acetone/dry ice). Then, TMS- $\mathrm{CHN}_{2}$ was added portion-wise to the mixture until the green colour of the starting thioketone disappeared. The magnetically stirred reaction mixture was allowed to warm slowly to $c a 268$ to $273 \mathrm{~K}$ and at this temperature a commercially available solution of tetrabutylammonium fluoride (TBAF, $1 \mathrm{ml}, 1 \mathrm{M}$ ) was added in small portions. Stirring was continued for $20 \mathrm{~min}$, and after warming to room temperature, the solvent was evaporated under vacuum. The crude product was analyzed by ${ }^{1} \mathrm{H}$ NMR spectroscopy, which revealed the presence of two isomeric ethenes in a ratio of $c a$ 10:1. After column chromatography $\left(\mathrm{SiO}_{2}, \mathrm{CH}_{2} \mathrm{Cl}_{2} /\right.$ hexane $3: 7)$, the major product was isolated, contaminated with a small admixture of the minor one, as an analytically pure sample (78\% yield). After additional crystallization from a hexane $/ \mathrm{CH}_{2} \mathrm{Cl}_{2}$ mixture, $285 \mathrm{mg}(54 \%)$ of pure $(E)-\mathbf{1}$ were isolated as orange crystals with m.p. 485-487 K. From this material, crystals suitable for the X-ray diffraction measurements were separated without additional recrystallization.
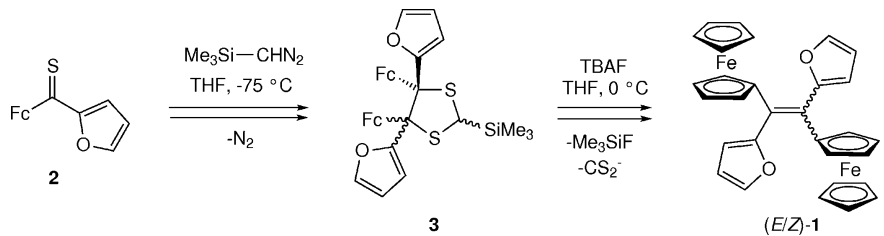

${ }^{1} \mathrm{H}$ NMR [600 MHz, $\left.\mathrm{CDCl}_{3}, \delta(\mathrm{ppm}), J(\mathrm{~Hz})\right]: 3.63-3.65$ $[m, 4 \mathrm{CH}(\mathrm{Fc})], 4.13-4.15[m, 4 \mathrm{CH}(\mathrm{Fc})], 4.16[s, 10 \mathrm{CH}(\mathrm{Fc})], 6.40$ $\left[d,{ }^{3} J_{\mathrm{H}, \mathrm{H}}=3.0,2 \mathrm{CH}\right.$ (Fur)], $6.54\left[d d,{ }^{4} J_{\mathrm{H}, \mathrm{H}}=1.8,{ }^{3} J_{\mathrm{H}, \mathrm{H}}=3.0\right.$, $2 \mathrm{CH}$ (Fur)], 7.58 [brs, 2CH(Fur)]. ${ }^{13} \mathrm{C} \mathrm{NMR} \mathrm{[150} \mathrm{MHz}, \mathrm{CDCl}_{3}$, 
Table 2

Experimental details.

\begin{tabular}{|c|c|}
\hline \multicolumn{2}{|l|}{ Crystal data } \\
\hline Chemical formula & {$\left[\mathrm{Fe}_{2}\left(\mathrm{C}_{5} \mathrm{H}_{5}\right)_{2}\left(\mathrm{C}_{20} \mathrm{H}_{14} \mathrm{O}_{2}\right)\right]$} \\
\hline$M_{\mathrm{r}}$ & 528.19 \\
\hline Crystal system, space group & Monoclinic, $P 2_{1} / n$ \\
\hline Temperature $(\mathrm{K})$ & 160 \\
\hline$a, b, c(\AA)$ & $\begin{array}{l}5.81006(13), 22.7138(5) \\
\quad 8.38031(18)\end{array}$ \\
\hline$\beta\left(^{\circ}\right)$ & $91.785(2)$ \\
\hline$V\left(\AA^{3}\right)$ & $1105.40(4)$ \\
\hline$Z$ & 2 \\
\hline Radiation type & Mo $K \alpha$ \\
\hline$\mu\left(\mathrm{mm}^{-1}\right)$ & 1.34 \\
\hline Crystal size $(\mathrm{mm})$ & $0.20 \times 0.16 \times 0.08$ \\
\hline \multicolumn{2}{|l|}{ Data collection } \\
\hline Diffractometer & $\begin{array}{l}\text { Oxford Diffraction SuperNova, } \\
\text { dual-radiation diffractometer }\end{array}$ \\
\hline Absorption correction & $\begin{array}{l}\text { Multi-scan (CrysAlis PRO; } \\
\text { Rigaku OD, 2015) }\end{array}$ \\
\hline$T_{\min }, T_{\max }$ & $0.895,1.000$ \\
\hline $\begin{array}{l}\text { No. of measured, independent and } \\
\text { observed }[I>2 \sigma(I)] \text { reflections }\end{array}$ & $13881,3021,2612$ \\
\hline$R_{\text {int }}$ & 0.026 \\
\hline$(\sin \theta / \lambda)_{\max }\left(\AA^{-1}\right)$ & 0.708 \\
\hline \multicolumn{2}{|l|}{ Refinement } \\
\hline$R\left[F^{2}>2 \sigma\left(F^{2}\right)\right], w R\left(F^{2}\right), S$ & $0.032,0.077,1.04$ \\
\hline No. of reflections & 3021 \\
\hline No. of parameters & 154 \\
\hline $\mathrm{H}$-atom treatment & $\mathrm{H}$-atom parameters constrained \\
\hline$\Delta \rho_{\max }, \Delta \rho_{\min }\left(\mathrm{e} \AA^{-3}\right)$ & $0.49,-0.36$ \\
\hline
\end{tabular}

Computer programs: CrysAlis PRO (Rigaku OD, 2015), SHELXT2014 (Sheldrick, 2015a), SHELXL2014 (Sheldrick, 2015b), ORTEPII (Johnson, 1976), Mercury (Macrae et al., 2006), PLATON (Spek, 2015) and publCIF (Westrip, 2010).

$\delta(\mathrm{ppm})]: 68.5,68.9$ [2 signals for $8 \mathrm{CH}(\mathrm{Fc})], 69.6$ [10CH( $\mathrm{Fc})]$, 85.4 [2C(Fc)], 109.1, 111.1, 140.8 [3 signals for $6 \mathrm{CH}(\mathrm{Fur})]$, $129.1(\mathrm{C}=\mathrm{C}), 153.3$ [2C(Fur)]. ESI-MS (mixture of isomers): $528\left(100,[M]^{+}\right), 529\left(50,[M+1]^{+}\right)$. Elemental analysis calculated for $\mathrm{C}_{30} \mathrm{H}_{24} \mathrm{Fe}_{2} \mathrm{O}_{2}$ (528.20): $\mathrm{C} 68.22, \mathrm{H} 4.58 \%$; found: $\mathrm{C}$ 68.38, H $4.61 \%$.

\section{Refinement}

Crystal data, data collection and structure refinement details are summarized in Table 2. All $\mathrm{H}$ atoms were placed in geometrically calculated positions and were constrained to ride on their parent atom with $\mathrm{C}-\mathrm{H}=0.95 \AA$ and with $U_{\text {iso }}(\mathrm{H})=1.2 U_{\text {eq }}(\mathrm{C})$.

\section{Acknowledgements}

GM thanks Professor Wolfgang Weigand (Jena) for stimulating discussions on the chemistry of ferrocene thioketones.

\section{Funding information}

Funding for this research was provided by: Maestro-3 (grant No. Dec-2012/06/A/ST5/00219 to R. Hamera-Fałdyga, G. Mlostoń); Institutspartnerschaft, Alexander von Humboldt Foundation, Bonn (grant to R. Hamera-Fałdyga, G. Mlostoń).

\section{References}

Astruc, D. (2017). Eur. J. Inorg. Chem. pp. 6-29.

Beletskaya, I. P., Tsvetkov, A. V., Latyshev, G. V., Tafeenko, V. A. \& Lukashev, N. V. (2001). J. Organomet. Chem. 637, 653-663.

Chen, Y. J., Pan, D.-S., Chiu, C.-F., Su, J.-X., Lin, S. J. \& Kwan, K. S. (2000). Inorg. Chem. 39, 953-958.

Dang, Y., Geise, H., Dommisse, R. \& Esmans, E. (1990). Inorg. Chim. Acta, 175, 115-120.

DeHope, A., Mendoza-Espinosa, D., Donnadieu, B. \& Bertrand, G. (2011). New J. Chem. 35, 2037-2042.

Denifl, P., Hradsky, A., Bildstein, B. \& Wurst, K. (1996). J. Organomet. Chem. 523, 79-91.

Dong, T.-Y., Ke, T.-J., Peng, S.-M. \& Yeh, S.-K. (1989). Inorg. Chem. 28, 2103-2106.

Farrugia, L. J., Evans, C., Lentz, D. \& Roemer, M. (2009). J. Am. Chem. Soc. 131, 1251-1268.

García, J. J. S., Flores-Alamo, M., Flores, D. E. C. \& Klimova, E. I. (2017). Mendeleev Commun. 27, 26-28.

García, J. J. S., Flores-Alamo, M., Ortiz-Frade, L. \& Klimova, E. I. (2017). J. Organomet. Chem. 842, 21-31.

García, J. J. S., Ortiz-Frade, L., Martínez-Klimova, E., García-Ramos, J. C., Flores-Alamo, M., Apan, T. R. \& Klimova, E. I. (2014). Open J. Synth. Theory Appl. 3, 44-56.

Groom, C. R., Bruno, I. J., Lightfoot, M. P. \& Ward, S. C. (2016). Acta Cryst. B72, 171-179.

Heigl, O. M., Herker, M. A., Hiller, W., Köhler, F. H. \& Schell, A. (1999). J. Organomet. Chem. 574, 94-98.

Jaouen, G., Vessières, A. \& Top, S. (2015). Chem. Soc. Rev. 44, 88028817.

Johnson, C. K. (1976). ORTEPII. Report ORNL-5138. Oak Ridge National Laboratory, Tennessee, USA.

Klimova, E. I., Berestneva, T. K., Cinquantini, A., Corsini, M., Zanello, P., Tuscano, R. A., Hernández-Ortega, S. \& MartínezGarcía, M. (2003). Org. Biomol. Chem. 1, 4458-4464.

Klimova, E. I., Berestneva, T. K., Ramirez, L. R., Cinquantini, A., Corsini, M., Zanello, P., Hernández-Ortega, S. \& García, M. M. (2003). Eur. J. Org. Chem. pp. 4265-4272.

Klimova, E. I., Flores-Alamo, M., Maya, S. C., García-Ramos, J. C., Ortiz-Frade, L. \& Stivalet, J. M. M. (2013). J. Organomet. Chem. 743, 24-30.

Klimova, E. I., Klimova, T., Flores-Alamo, M., Backinowsky, L. V. \& García, M. M. (2009). Molecules, 14, 3161-3175.

Macrae, C. F., Edgington, P. R., McCabe, P., Pidcock, E., Shields, G. P., Taylor, R., Towler, M. \& van de Streek, J. (2006). J. Appl. Cryst. 39, 453-457.

Mata, J. A. \& Peris, E. (2001). J. Chem. Soc. Dalton Trans. pp. 3634 3640.

Mlostoń, G., Hamera, R. \& Heimgartner, H. (2015). Phosphorus Sulfur Silicon Relat. Elem. 190, 2125-2133.

Mlostoń, G., Hamera-Fałdyga, R. \& Heimgartner, H. (2018). J. Sulfur Chem. 39, doi: 10.1080/17415993.2017.1415339

Mlostoń, G., Pipiak, P., Hamera-Fałdyga, R. \& Heimgartner, H. (2017). Beilstein J. Org. Chem. 13, 1900-1906.

Nagahora, N., Yuasa, A., Sasamori, T. \& Tokitoh, N. (2007). Acta Cryst. E63, m2702.

Resnier, R., Galopin, N., Sibiril, Y., Clavreul, A., Cayon, J., Briganti, A., Legras, P., Vessières, A., Montier, T., Jaouen, G., Benoit, J.-P. \& Passirani, C. (2017). Pharmacol. Res. 126, 54-65.

Rigaku OD (2015). CrysAlis PRO. Rigaku Oxford Diffraction, Abingdon, England.

Roemer, M., Donnadieu, B. \& Nijhuis, C. A. (2016). Eur. J. Inorg. Chem. pp. 1314-1318.

Roemer, M. \& Lentz, D. (2008). Eur. J. Inorg. Chem. pp. 4875-4878.

Sheldrick, G. M. (2015a). Acta Cryst. A71, 3-8.

Sheldrick, G. M. (2015b). Acta Cryst. C71, 3-8.

Skibar, W., Kopacka, H., Wurst, K., Salzmann, C., Ongania, K.-H., de Biani, F. F., Zanello, P. \& Bildstein, B. (2004). Organometallics, 23, 1024-1041. 
Solntsev, P. V., Dudkin, S. V., Sabin, J. R. \& Nemykin, V. N. (2011). Organometallics, 30, 3037-3046.

Spek, A. L. (2015). Acta Cryst. C71, 9-18.
Top, S., Dauer, B., Vaissermann, J. \& Jaouen, G. (1997). J. Organomet. Chem. 541, 355-361.

Westrip, S. P. (2010). J. Appl. Cryst. 43, 920-925. 


\section{supporting information}

Acta Cryst. (2018). E74, 625-629 [https://doi.org/10.1107/S2056989018005078]

Crystal structure of $(E)-1,2-$ diferrocenyl-1,2-bis(furan-2-yl) ethene

Anthony Linden, Róża Hamera-Fałdyga, Grzegorz Mlostoń and Heinz Heimgartner

Computing details

Data collection: CrysAlis PRO (Rigaku OD, 2015); cell refinement: CrysAlis PRO (Rigaku OD, 2015); data reduction: CrysAlis PRO (Rigaku OD, 2015); program(s) used to solve structure: SHELXT2014 (Sheldrick, 2015a); program(s) used to refine structure: SHELXL2014 (Sheldrick, 2015b); molecular graphics: ORTEPII (Johnson, 1976) and Mercury (Macrae et al., 2006); software used to prepare material for publication: SHELXL2014 (Sheldrick, 2015b), PLATON (Spek, 2015) and publCIF (Westrip, 2010).

(E)-1,2-Diferrocenyl-1,2-bis(furan-2-yl)ethene

Crystal data

$\left[\mathrm{Fe}_{2}\left(\mathrm{C}_{5} \mathrm{H}_{5}\right)_{2}\left(\mathrm{C}_{20} \mathrm{H}_{14} \mathrm{O}_{2}\right)\right]$

$M_{r}=528.19$

Monoclinic, $P 2{ }_{1} / n$

$a=5.81006(13) \AA$

$b=22.7138(5) \AA$

$c=8.38031(18) \AA$

$\beta=91.785(2)^{\circ}$

$V=1105.40(4) \AA^{3}$

$Z=2$

Data collection

Oxford Diffraction SuperNova, dual radiation diffractometer

Radiation source: SuperNova (Mo) X-ray Source

Mirror monochromator

Detector resolution: 10.3801 pixels $\mathrm{mm}^{-1}$

$\omega$ scans

Absorption correction: multi-scan

(CrysAlisPro; Rigaku OD, 2015)

\section{Refinement}

Refinement on $F^{2}$

Least-squares matrix: full

$R\left[F^{2}>2 \sigma\left(F^{2}\right)\right]=0.032$

$w R\left(F^{2}\right)=0.077$

$S=1.04$

3021 reflections

154 parameters

0 restraints
$F(000)=544$

$D_{\mathrm{x}}=1.587 \mathrm{Mg} \mathrm{m}^{-3}$

Mo $K \alpha$ radiation, $\lambda=0.71073 \AA$

Cell parameters from 7365 reflections

$\theta=3.0-29.9^{\circ}$

$\mu=1.34 \mathrm{~mm}^{-1}$

$T=160 \mathrm{~K}$

Tablet, orange

$0.20 \times 0.16 \times 0.08 \mathrm{~mm}$

$T_{\min }=0.895, T_{\max }=1.000$

13881 measured reflections

3021 independent reflections

2612 reflections with $I>2 \sigma(I)$

$R_{\text {int }}=0.026$

$\theta_{\max }=30.2^{\circ}, \theta_{\min }=2.6^{\circ}$

$h=-8 \rightarrow 7$

$k=-31 \rightarrow 31$

$l=-11 \rightarrow 11$

Hydrogen site location: inferred from neighbouring sites

$\mathrm{H}$-atom parameters constrained

$w=1 /\left[\sigma^{2}\left(F_{\mathrm{o}}^{2}\right)+(0.0291 P)^{2}+0.9916 P\right]$

where $P=\left(F_{\mathrm{o}}^{2}+2 F_{\mathrm{c}}^{2}\right) / 3$

$(\Delta / \sigma)_{\max }=0.001$

$\Delta \rho_{\max }=0.49 \mathrm{e} \AA^{-3}$

$\Delta \rho_{\min }=-0.36 \mathrm{e} \AA^{-3}$ 


\section{Special details}

Experimental. Data collection and full structure determination done by Prof. Anthony Linden: anthony.linden@chem.uzh.ch

Solvent used: hexane / dichloromethane Cooling Device: Oxford Instruments Cryojet XL Crystal mount: on a glass fibre Frames collected: 1290 Seconds exposure per frame: 10.0 Degrees rotation per frame: 1602.0 Crystal-detector distance (mm): 55.0

Geometry. All esds (except the esd in the dihedral angle between two 1.s. planes) are estimated using the full covariance matrix. The cell esds are taken into account individually in the estimation of esds in distances, angles and torsion angles; correlations between esds in cell parameters are only used when they are defined by crystal symmetry. An approximate (isotropic) treatment of cell esds is used for estimating esds involving 1.s. planes.

Fractional atomic coordinates and isotropic or equivalent isotropic displacement parameters $\left(\AA^{2}\right)$

\begin{tabular}{lllll}
\hline & $x$ & $y$ & $z$ & $U_{\text {iso }} / U_{\text {eq }}$ \\
\hline Fe1 & $0.46176(4)$ & $0.65950(2)$ & $0.41357(3)$ & $0.01767(8)$ \\
O1 & $0.5980(2)$ & $0.54191(6)$ & $0.79034(16)$ & $0.0295(3)$ \\
C1 & $0.5597(3)$ & $0.52572(7)$ & $0.5053(2)$ & $0.0159(3)$ \\
C2 & $0.7063(3)$ & $0.53842(7)$ & $0.6485(2)$ & $0.0187(3)$ \\
C3 & $0.7668(5)$ & $0.55207(10)$ & $0.9050(3)$ & $0.0408(6)$ \\
H3 & 0.7408 & 0.5562 & 1.0158 & $0.049^{*}$ \\
C4 & $0.9717(4)$ & $0.55529(10)$ & $0.8395(3)$ & $0.0418(6)$ \\
H4 & 1.1150 & 0.5624 & 0.8938 & $0.050^{*}$ \\
C5 & $0.9354(3)$ & $0.54589(8)$ & $0.6699(2)$ & $0.0256(4)$ \\
H5 & 1.0487 & 0.5451 & 0.5906 & $0.031^{*}$ \\
C6 & $0.5501(3)$ & $0.57204(7)$ & $0.38168(19)$ & $0.0168(3)$ \\
C7 & $0.7351(3)$ & $0.61094(7)$ & $0.3435(2)$ & $0.0212(3)$ \\
H7 & 0.8841 & 0.6111 & 0.3937 & $0.025^{*}$ \\
C8 & $0.6590(4)$ & $0.64909(8)$ & $0.2187(2)$ & $0.0264(4)$ \\
H8 & 0.7483 & 0.6788 & 0.1701 & $0.032^{*}$ \\
C9 & $0.4265(4)$ & $0.63519(8)$ & $0.1791(2)$ & $0.0262(4)$ \\
H9 & 0.3324 & 0.6541 & 0.0998 & $0.031^{*}$ \\
C10 & $0.3583(3)$ & $0.58793(7)$ & $0.2787(2)$ & $0.0200(3)$ \\
H10 & 0.2106 & 0.5700 & 0.2772 & $0.024^{*}$ \\
C11 & $0.4528(4)$ & $0.68024(9)$ & $0.6520(2)$ & $0.0295(4)$ \\
H11 & 0.5075 & 0.6563 & 0.7382 & $0.035^{*}$ \\
C12 & $0.5838(3)$ & $0.72260(9)$ & $0.5685(2)$ & $0.0310(4)$ \\
H12 & 0.7412 & 0.7320 & 0.5891 & $0.037^{*}$ \\
C13 & $0.4377(4)$ & $0.74812(8)$ & $0.4493(2)$ & $0.0286(4)$ \\
H13 & 0.4798 & 0.7778 & 0.3758 & $0.034^{*}$ \\
C14 & $0.2176(3)$ & $0.72175(8)$ & $0.4587(2)$ & $0.0267(4)$ \\
H14 & 0.0866 & 0.7305 & 0.3922 & $0.032^{*}$ \\
C15 & $0.2268(3)$ & $0.68009(8)$ & $0.5840(2)$ & $0.0268(4)$ \\
H15 & 0.1028 & 0.6562 & 0.6169 & $0.032^{*}$ \\
& & & &
\end{tabular}

Atomic displacement parameters $\left(\AA^{2}\right)$

\begin{tabular}{lllllll}
\hline & $U^{11}$ & $U^{22}$ & $U^{33}$ & $U^{12}$ & $U^{13}$ & $U^{23}$ \\
\hline $\mathrm{Fe} 1$ & $0.02204(13)$ & $0.01261(12)$ & $0.01843(13)$ & $0.00067(9)$ & $0.00192(9)$ & $-0.00138(9)$ \\
$\mathrm{O} 1$ & $0.0359(8)$ & $0.0287(7)$ & $0.0239(7)$ & $0.0074(6)$ & $0.0035(6)$ & $-0.0018(6)$
\end{tabular}




$\begin{array}{lllllll}\text { C1 } & 0.0146(7) & 0.0145(7) & 0.0186(8) & 0.0026(6) & 0.0019(6) & -0.0016(6) \\ \text { C2 } & 0.0225(8) & 0.0125(7) & 0.0211(8) & 0.0001(6) & 0.0004(6) & -0.0008(6) \\ \text { C3 } & 0.0646(16) & 0.0329(11) & 0.0242(10) & 0.0080(11) & -0.0104(10) & -0.0070(9) \\ \text { C4 } & 0.0466(13) & 0.0289(11) & 0.0480(13) & -0.0068(10) & -0.0295(11) & 0.0027(10) \\ \text { C5 } & 0.0207(8) & 0.0236(9) & 0.0324(10) & -0.0016(7) & -0.0022(7) & 0.0050(8) \\ \text { C6 } & 0.0192(8) & 0.0130(7) & 0.0184(8) & 0.0008(6) & 0.0028(6) & -0.0025(6) \\ \text { C7 } & 0.0214(8) & 0.0167(8) & 0.0260(9) & 0.0003(6) & 0.0064(7) & -0.0014(7) \\ \text { C8 } & 0.0377(10) & 0.0189(8) & 0.0233(9) & -0.0002(7) & 0.0127(8) & 0.0012(7) \\ \text { C9 } & 0.0414(11) & 0.0192(8) & 0.0179(8) & 0.0057(8) & -0.0011(7) & -0.0012(7) \\ \text { C10 } & 0.0242(8) & 0.0153(8) & 0.0204(8) & 0.0020(6) & -0.0022(7) & -0.0037(6) \\ \text { C11 } & 0.0464(12) & 0.0227(9) & 0.0193(8) & 0.0082(8) & -0.0015(8) & -0.0057(7) \\ \text { C12 } & 0.0278(10) & 0.0274(10) & 0.0378(11) & -0.0027(8) & 0.0019(8) & -0.0172(8) \\ \text { C13 } & 0.0407(11) & 0.0133(8) & 0.0324(10) & 0.0001(7) & 0.0121(8) & -0.0032(7) \\ \text { C14 } & 0.0282(9) & 0.0233(9) & 0.0285(9) & 0.0079(7) & 0.0006(8) & -0.0050(7) \\ \text { C15 } & 0.0326(10) & 0.0219(9) & 0.0267(9) & -0.0035(7) & 0.0116(8) & -0.0063(7)\end{array}$

Geometric parameters $\left(\AA,{ }^{\circ}\right)$

\begin{tabular}{|c|c|c|c|}
\hline $\mathrm{Fe} 1-\mathrm{C} 7$ & $2.0352(17)$ & $\mathrm{C} 5-\mathrm{H} 5$ & 0.9500 \\
\hline $\mathrm{Fe} 1-\mathrm{C} 8$ & $2.0380(18)$ & $\mathrm{C} 6-\mathrm{C} 10$ & $1.434(2)$ \\
\hline $\mathrm{Fe} 1-\mathrm{C} 13$ & $2.0404(18)$ & $\mathrm{C} 6-\mathrm{C} 7$ & $1.435(2)$ \\
\hline $\mathrm{Fe} 1-\mathrm{C} 9$ & $2.0456(18)$ & $\mathrm{C} 7-\mathrm{C} 8$ & $1.419(3)$ \\
\hline $\mathrm{Fe} 1-\mathrm{C} 12$ & $2.0457(19)$ & $\mathrm{C} 7-\mathrm{H} 7$ & 0.9500 \\
\hline $\mathrm{Fe} 1-\mathrm{C} 14$ & $2.0465(18)$ & $\mathrm{C} 8-\mathrm{C} 9$ & $1.417(3)$ \\
\hline $\mathrm{Fe} 1-\mathrm{C} 11$ & $2.0555(19)$ & $\mathrm{C} 8-\mathrm{H} 8$ & 0.9500 \\
\hline $\mathrm{Fe} 1-\mathrm{C} 10$ & $2.0591(17)$ & $\mathrm{C} 9-\mathrm{C} 10$ & $1.424(3)$ \\
\hline $\mathrm{Fe} 1-\mathrm{C} 15$ & $2.0605(18)$ & C9-H9 & 0.9500 \\
\hline $\mathrm{Fe} 1-\mathrm{C} 6$ & $2.0712(16)$ & $\mathrm{C} 10-\mathrm{H} 10$ & 0.9500 \\
\hline $\mathrm{O} 1-\mathrm{C} 2$ & $1.365(2)$ & $\mathrm{C} 11-\mathrm{C} 15$ & $1.415(3)$ \\
\hline $\mathrm{O} 1-\mathrm{C} 3$ & $1.371(3)$ & $\mathrm{C} 11-\mathrm{C} 12$ & $1.424(3)$ \\
\hline $\mathrm{C} 1-\mathrm{C} 1^{\mathrm{i}}$ & $1.360(3)$ & $\mathrm{C} 11-\mathrm{H} 11$ & 0.9500 \\
\hline $\mathrm{C} 1-\mathrm{C} 6$ & $1.476(2)$ & $\mathrm{C} 12-\mathrm{C} 13$ & $1.415(3)$ \\
\hline $\mathrm{C} 1-\mathrm{C} 2$ & $1.478(2)$ & $\mathrm{C} 12-\mathrm{H} 12$ & 0.9500 \\
\hline $\mathrm{C} 2-\mathrm{C} 5$ & $1.349(2)$ & $\mathrm{C} 13-\mathrm{C} 14$ & $1.416(3)$ \\
\hline $\mathrm{C} 3-\mathrm{C} 4$ & $1.329(4)$ & $\mathrm{C} 13-\mathrm{H} 13$ & 0.9500 \\
\hline $\mathrm{C} 3-\mathrm{H} 3$ & 0.9500 & $\mathrm{C} 14-\mathrm{C} 15$ & $1.414(3)$ \\
\hline $\mathrm{C} 4-\mathrm{C} 5$ & $1.446(3)$ & $\mathrm{C} 14-\mathrm{H} 14$ & 0.9500 \\
\hline $\mathrm{C} 4-\mathrm{H} 4$ & 0.9500 & $\mathrm{C} 15-\mathrm{H} 15$ & 0.9500 \\
\hline $\mathrm{C} 7-\mathrm{Fe} 1-\mathrm{C} 8$ & $40.77(7)$ & $\mathrm{C} 10-\mathrm{C} 6-\mathrm{C} 7$ & $106.52(15)$ \\
\hline $\mathrm{C} 7-\mathrm{Fe} 1-\mathrm{C} 13$ & $129.40(8)$ & $\mathrm{C} 10-\mathrm{C} 6-\mathrm{C} 1$ & $127.83(15)$ \\
\hline $\mathrm{C} 8-\mathrm{Fe} 1-\mathrm{C} 13$ & $105.96(8)$ & $\mathrm{C} 7-\mathrm{C} 6-\mathrm{C} 1$ & $125.64(15)$ \\
\hline $\mathrm{C} 7-\mathrm{Fe} 1-\mathrm{C} 9$ & $68.43(8)$ & $\mathrm{C} 10-\mathrm{C} 6-\mathrm{Fe} 1$ & $69.23(9)$ \\
\hline $\mathrm{C} 8-\mathrm{Fe} 1-\mathrm{C} 9$ & $40.59(8)$ & $\mathrm{C} 7-\mathrm{C} 6-\mathrm{Fe} 1$ & $68.20(9)$ \\
\hline $\mathrm{C} 13-\mathrm{Fe} 1-\mathrm{C} 9$ & $113.70(8)$ & $\mathrm{C} 1-\mathrm{C} 6-\mathrm{Fe} 1$ & $126.65(11)$ \\
\hline $\mathrm{C} 7-\mathrm{Fe} 1-\mathrm{C} 12$ & $107.73(8)$ & $\mathrm{C} 8-\mathrm{C} 7-\mathrm{C} 6$ & $108.76(16)$ \\
\hline $\mathrm{C} 8-\mathrm{Fe} 1-\mathrm{C} 12$ & $113.42(8)$ & $\mathrm{C} 8-\mathrm{C} 7-\mathrm{Fe} 1$ & $69.73(10)$ \\
\hline $\mathrm{C} 13-\mathrm{Fe} 1-\mathrm{C} 12$ & $40.51(8)$ & $\mathrm{C} 6-\mathrm{C} 7-\mathrm{Fe} 1$ & $70.89(9)$ \\
\hline
\end{tabular}




$$
\begin{aligned}
& \text { C9-Fe1- } 12 \\
& \mathrm{C} 7-\mathrm{Fe} 1-\mathrm{C} 14 \\
& \mathrm{C} 8-\mathrm{Fe} 1-\mathrm{C} 14 \\
& \text { C13-Fe1-C14 } \\
& \text { C9-Fe1-C14 } \\
& \mathrm{C} 12-\mathrm{Fe} 1-\mathrm{C} 14 \\
& \mathrm{C} 7-\mathrm{Fe} 1-\mathrm{C} 11 \\
& \mathrm{C} 8-\mathrm{Fe} 1-\mathrm{C} 11 \\
& \mathrm{C} 13-\mathrm{Fe} 1-\mathrm{C} 11 \\
& \text { C9-Fe1-C11 } \\
& \mathrm{C} 12-\mathrm{Fe} 1-\mathrm{C} 11 \\
& \mathrm{C} 14-\mathrm{Fe} 1-\mathrm{C} 11 \\
& \mathrm{C} 7-\mathrm{Fe} 1-\mathrm{C} 10 \\
& \mathrm{C} 8-\mathrm{Fe} 1-\mathrm{C} 10 \\
& \mathrm{C} 13-\mathrm{Fe} 1-\mathrm{C} 10 \\
& \text { C9-Fe1-C10 } \\
& \mathrm{C} 12-\mathrm{Fe} 1-\mathrm{C} 10 \\
& \mathrm{C} 14-\mathrm{Fe} 1-\mathrm{C} 10 \\
& \mathrm{C} 11-\mathrm{Fe} 1-\mathrm{C} 10 \\
& \mathrm{C} 7-\mathrm{Fe} 1-\mathrm{C} 15 \\
& \mathrm{C} 8-\mathrm{Fe} 1-\mathrm{C} 15 \\
& \mathrm{C} 13-\mathrm{Fe} 1-\mathrm{C} 15 \\
& \mathrm{C} 9-\mathrm{Fe} 1-\mathrm{C} 15 \\
& \mathrm{C} 12-\mathrm{Fe} 1-\mathrm{C} 15 \\
& \mathrm{C} 14-\mathrm{Fe} 1-\mathrm{C} 15 \\
& \mathrm{C} 11-\mathrm{Fe} 1-\mathrm{C} 15 \\
& \mathrm{C} 10-\mathrm{Fe} 1-\mathrm{C} 15 \\
& \mathrm{C} 7-\mathrm{Fe} 1-\mathrm{C} 6 \\
& \mathrm{C} 8-\mathrm{Fe} 1-\mathrm{C} 6 \\
& \mathrm{C} 13-\mathrm{Fe} 1-\mathrm{C} 6 \\
& \text { C9-Fe1-C6 } \\
& \text { C12-Fe1-C6 } \\
& \mathrm{C} 14-\mathrm{Fe} 1-\mathrm{C} 6 \\
& \text { C11-Fe1-C6 } \\
& \mathrm{C} 10-\mathrm{Fe} 1-\mathrm{C} 6 \\
& \mathrm{C} 15-\mathrm{Fe} 1-\mathrm{C} 6 \\
& \mathrm{C} 2-\mathrm{O} 1-\mathrm{C} 3 \\
& \mathrm{C} 1{ }^{\mathrm{i}}-\mathrm{C} 1-\mathrm{C} 6 \\
& \mathrm{C} 1{ }^{\mathrm{i}}-\mathrm{C} 1-\mathrm{C} 2 \\
& \mathrm{C} 6-\mathrm{C} 1-\mathrm{C} 2 \\
& \mathrm{C} 5-\mathrm{C} 2-\mathrm{O} 1 \\
& \mathrm{C} 5-\mathrm{C} 2-\mathrm{C} 1 \\
& \mathrm{O} 1-\mathrm{C} 2-\mathrm{C} 1 \\
& \text { C4- } 3 \text { - }-\mathrm{O} 1 \\
& \mathrm{C} 4-\mathrm{C} 3-\mathrm{H} 3 \\
& \mathrm{O} 1-\mathrm{C} 3-\mathrm{H} 3 \\
& \mathrm{C} 3-\mathrm{C} 4-\mathrm{C} 5 \\
& \mathrm{C} 3-\mathrm{C} 4-\mathrm{H} 4
\end{aligned}
$$

$145.21(8)$
$168.36(8)$
$129.68(8)$
$40.56(8)$
$108.34(8)$
$68.12(8)$
$116.63(8)$
$146.90(9)$
$68.12(8)$
$172.39(9)$
$40.64(8)$
$67.88(8)$
$68.35(7)$
$68.30(7)$
$147.05(8)$
$40.58(7)$
$172.33(8)$
$117.03(7)$
$134.35(8)$
$149.65(8)$
$169.52(8)$
$67.94(8)$
$132.74(8)$
$67.98(8)$
$40.27(8)$
$40.22(8)$
$111.80(8)$
$40.91(7)$
$68.75(7)$
$169.58(8)$
$68.58(7)$
$132.19(8)$
$149.60(7)$
$111.05(7)$
$40.64(6)$
$118.64(7)$
$106.35(17)$
$123.93(19)$
$120.04(19)$
$116.02(14)$
$110.90(16)$
$132.40(16)$
$116.67(15)$
$110.49(19)$
124.8
124.8
$107.03(18)$
126.5

$\mathrm{C} 8-\mathrm{C} 7-\mathrm{H} 7$

$\mathrm{C} 6-\mathrm{C} 7-\mathrm{H} 7$

$\mathrm{Fe} 1-\mathrm{C} 7-\mathrm{H} 7$

C9- 8 8 $-\mathrm{C} 7$

$\mathrm{C} 9-\mathrm{C} 8-\mathrm{Fe} 1$

$\mathrm{C} 7-\mathrm{C} 8-\mathrm{Fe} 1$

$\mathrm{C} 9-\mathrm{C} 8-\mathrm{H} 8$

$\mathrm{C} 7-\mathrm{C} 8-\mathrm{H} 8$

$\mathrm{Fe} 1-\mathrm{C} 8-\mathrm{H} 8$

$\mathrm{C} 8-\mathrm{C} 9-\mathrm{C} 10$

$\mathrm{C} 8-\mathrm{C} 9-\mathrm{Fe} 1$

$\mathrm{C} 10-\mathrm{C} 9-\mathrm{Fe} 1$

$\mathrm{C} 8-\mathrm{C} 9-\mathrm{H} 9$

C10- $99-\mathrm{H} 9$

$\mathrm{Fe} 1-\mathrm{C} 9-\mathrm{H} 9$

C9- $10-\mathrm{C} 6$

C9- $\mathrm{C} 10-\mathrm{Fe} 1$

C6- $\mathrm{C} 10-\mathrm{Fe} 1$

C9- $\mathrm{C} 10-\mathrm{H} 10$

C6- $\mathrm{C} 10-\mathrm{H} 10$

$\mathrm{Fe} 1-\mathrm{C} 10-\mathrm{H} 10$

$\mathrm{C} 15-\mathrm{C} 11-\mathrm{C} 12$

$\mathrm{C} 15-\mathrm{C} 11-\mathrm{Fe} 1$

$\mathrm{C} 12-\mathrm{C} 11-\mathrm{Fe} 1$

C15-C11-H11

C12-C11-H11

$\mathrm{Fe} 1-\mathrm{C} 11-\mathrm{H} 11$

$\mathrm{C} 13-\mathrm{C} 12-\mathrm{C} 11$

$\mathrm{C} 13-\mathrm{C} 12-\mathrm{Fe} 1$

$\mathrm{C} 11-\mathrm{C} 12-\mathrm{Fe} 1$

$\mathrm{C} 13-\mathrm{C} 12-\mathrm{H} 12$

$\mathrm{C} 11-\mathrm{C} 12-\mathrm{H} 12$

$\mathrm{Fe} 1-\mathrm{C} 12-\mathrm{H} 12$

C12-C13-C14

$\mathrm{C} 12-\mathrm{C} 13-\mathrm{Fe} 1$

$\mathrm{C} 14-\mathrm{C} 13-\mathrm{Fe} 1$

$\mathrm{C} 12-\mathrm{C} 13-\mathrm{H} 13$

C14-C13-H13

$\mathrm{Fe} 1-\mathrm{C} 13-\mathrm{H} 13$

$\mathrm{C} 15-\mathrm{C} 14-\mathrm{C} 13$

$\mathrm{C} 15-\mathrm{C} 14-\mathrm{Fe} 1$

$\mathrm{C} 13-\mathrm{C} 14-\mathrm{Fe} 1$

C15-C14-H14

C13-C14-H14

$\mathrm{Fe} 1-\mathrm{C} 14-\mathrm{H} 14$

C14-C15-C11

$\mathrm{C} 14-\mathrm{C} 15-\mathrm{Fe} 1$

$\mathrm{C} 11-\mathrm{C} 15-\mathrm{Fe} 1$
125.6

125.6

125.3

108.05 (16)

69.99 (11)

$69.51(10)$

126.0

126.0

126.1

108.15 (16)

69.42 (10)

$70.22(10)$

125.9

125.9

126.0

$108.50(16)$

69.20 (10)

$70.13(9)$

125.8

125.8

126.5

$107.88(18)$

70.08 (11)

69.31 (11)

126.1

126.1

126.1

$107.82(18)$

69.54 (11)

70.05 (11)

126.1

126.1

125.9

108.09 (17)

69.94 (11)

$69.95(11)$

126.0

126.0

125.7

108.12 (17)

70.40 (11)

69.49 (11)

125.9

125.9

125.7

$108.10(17)$

$69.33(10)$

69.70 (11) 


\begin{tabular}{|c|c|c|c|}
\hline $\mathrm{C} 5-\mathrm{C} 4-\mathrm{H} 4$ & 126.5 & $\mathrm{C} 14-\mathrm{C} 15-\mathrm{H} 15$ & 126.0 \\
\hline $\mathrm{C} 2-\mathrm{C} 5-\mathrm{C} 4$ & $105.22(18)$ & $\mathrm{C} 11-\mathrm{C} 15-\mathrm{H} 15$ & 126.0 \\
\hline $\mathrm{C} 2-\mathrm{C} 5-\mathrm{H} 5$ & 127.4 & $\mathrm{Fe} 1-\mathrm{C} 15-\mathrm{H} 15$ & 126.6 \\
\hline $\mathrm{C} 4-\mathrm{C} 5-\mathrm{H} 5$ & 127.4 & & \\
\hline $\mathrm{C} 3-\mathrm{O} 1-\mathrm{C} 2-\mathrm{C} 5$ & $0.1(2)$ & $\mathrm{Fe} 1-\mathrm{C} 8-\mathrm{C} 9-\mathrm{C} 10$ & $-59.76(12)$ \\
\hline $\mathrm{C} 3-\mathrm{O} 1-\mathrm{C} 2-\mathrm{C} 1$ & $178.20(15)$ & $\mathrm{C} 7-\mathrm{C} 8-\mathrm{C} 9-\mathrm{Fe} 1$ & $59.30(12)$ \\
\hline $\mathrm{C} 1-\mathrm{C} 1-\mathrm{C} 2-\mathrm{C} 5$ & $115.8(2)$ & $\mathrm{C} 8-\mathrm{C} 9-\mathrm{C} 10-\mathrm{C} 6$ & $0.0(2)$ \\
\hline $\mathrm{C} 6-\mathrm{C} 1-\mathrm{C} 2-\mathrm{C} 5$ & $-65.4(2)$ & $\mathrm{Fe} 1-\mathrm{C} 9-\mathrm{C} 10-\mathrm{C} 6$ & $-59.30(12)$ \\
\hline $\mathrm{C} 1-\mathrm{C} 1-\mathrm{C} 2-\mathrm{O} 1$ & $-61.8(2)$ & $\mathrm{C} 8-\mathrm{C} 9-\mathrm{C} 10-\mathrm{Fe} 1$ & $59.26(13)$ \\
\hline $\mathrm{C} 6-\mathrm{C} 1-\mathrm{C} 2-\mathrm{O} 1$ & $117.02(16)$ & $\mathrm{C} 7-\mathrm{C} 6-\mathrm{C} 10-\mathrm{C} 9$ & $0.52(19)$ \\
\hline $\mathrm{C} 2-\mathrm{O} 1-\mathrm{C} 3-\mathrm{C} 4$ & $0.4(2)$ & $\mathrm{C} 1-\mathrm{C} 6-\mathrm{C} 10-\mathrm{C} 9$ & $179.64(16)$ \\
\hline $\mathrm{O} 1-\mathrm{C} 3-\mathrm{C} 4-\mathrm{C} 5$ & $-0.7(3)$ & $\mathrm{Fe} 1-\mathrm{C} 6-\mathrm{C} 10-\mathrm{C} 9$ & $58.72(12)$ \\
\hline $\mathrm{O} 1-\mathrm{C} 2-\mathrm{C} 5-\mathrm{C} 4$ & $-0.5(2)$ & $\mathrm{C} 7-\mathrm{C} 6-\mathrm{C} 10-\mathrm{Fe} 1$ & $-58.21(11)$ \\
\hline $\mathrm{C} 1-\mathrm{C} 2-\mathrm{C} 5-\mathrm{C} 4$ & $-178.21(18)$ & $\mathrm{C} 1-\mathrm{C} 6-\mathrm{C} 10-\mathrm{Fe} 1$ & $120.92(17)$ \\
\hline $\mathrm{C} 3-\mathrm{C} 4-\mathrm{C} 5-\mathrm{C} 2$ & $0.7(2)$ & $\mathrm{C} 15-\mathrm{C} 11-\mathrm{C} 12-\mathrm{C} 13$ & $-0.2(2)$ \\
\hline $\mathrm{C} 1{ }^{\mathrm{i}}-\mathrm{C} 1-\mathrm{C} 6-\mathrm{C} 10$ & $32.9(3)$ & $\mathrm{Fe} 1-\mathrm{C} 11-\mathrm{C} 12-\mathrm{C} 13$ & $59.52(13)$ \\
\hline $\mathrm{C} 2-\mathrm{C} 1-\mathrm{C} 6-\mathrm{C} 10$ & $-145.89(16)$ & $\mathrm{C} 15-\mathrm{C} 11-\mathrm{C} 12-\mathrm{Fe} 1$ & $-59.70(13)$ \\
\hline $\mathrm{C} 1-\mathrm{C} 1-\mathrm{C} 6-\mathrm{C} 7$ & $-148.2(2)$ & $\mathrm{C} 11-\mathrm{C} 12-\mathrm{C} 13-\mathrm{C} 14$ & $-0.1(2)$ \\
\hline $\mathrm{C} 2-\mathrm{C} 1-\mathrm{C} 6-\mathrm{C} 7$ & $33.1(2)$ & $\mathrm{Fe} 1-\mathrm{C} 12-\mathrm{C} 13-\mathrm{C} 14$ & $59.79(13)$ \\
\hline $\mathrm{C} 1-\mathrm{C} 1-\mathrm{C} 6-\mathrm{Fe} 1$ & $123.9(2)$ & $\mathrm{C} 11-\mathrm{C} 12-\mathrm{C} 13-\mathrm{Fe} 1$ & $-59.84(13)$ \\
\hline $\mathrm{C} 2-\mathrm{C} 1-\mathrm{C} 6-\mathrm{Fe} 1$ & $-54.85(19)$ & $\mathrm{C} 12-\mathrm{C} 13-\mathrm{C} 14-\mathrm{C} 15$ & $0.3(2)$ \\
\hline $\mathrm{C} 10-\mathrm{C} 6-\mathrm{C} 7-\mathrm{C} 8$ & $-0.81(19)$ & $\mathrm{Fe} 1-\mathrm{C} 13-\mathrm{C} 14-\mathrm{C} 15$ & $60.05(13)$ \\
\hline $\mathrm{C} 1-\mathrm{C} 6-\mathrm{C} 7-\mathrm{C} 8$ & $-179.96(15)$ & $\mathrm{C} 12-\mathrm{C} 13-\mathrm{C} 14-\mathrm{Fe} 1$ & $-59.78(13)$ \\
\hline $\mathrm{Fe} 1-\mathrm{C} 6-\mathrm{C} 7-\mathrm{C} 8$ & $-59.67(12)$ & $\mathrm{C} 13-\mathrm{C} 14-\mathrm{C} 15-\mathrm{C} 11$ & $-0.4(2)$ \\
\hline $\mathrm{C} 10-\mathrm{C} 6-\mathrm{C} 7-\mathrm{Fe} 1$ & $58.86(11)$ & $\mathrm{Fe} 1-\mathrm{C} 14-\mathrm{C} 15-\mathrm{C} 11$ & $59.11(13)$ \\
\hline $\mathrm{C} 1-\mathrm{C} 6-\mathrm{C} 7-\mathrm{Fe} 1$ & $-120.29(16)$ & $\mathrm{C} 13-\mathrm{C} 14-\mathrm{C} 15-\mathrm{Fe} 1$ & $-59.48(13)$ \\
\hline $\mathrm{C} 6-\mathrm{C} 7-\mathrm{C} 8-\mathrm{C} 9$ & $0.8(2)$ & $\mathrm{C} 12-\mathrm{C} 11-\mathrm{C} 15-\mathrm{C} 14$ & $0.3(2)$ \\
\hline $\mathrm{Fe} 1-\mathrm{C} 7-\mathrm{C} 8-\mathrm{C} 9$ & $-59.60(13)$ & $\mathrm{Fe} 1-\mathrm{C} 11-\mathrm{C} 15-\mathrm{C} 14$ & $-58.88(13)$ \\
\hline $\mathrm{C} 6-\mathrm{C} 7-\mathrm{C} 8-\mathrm{Fe} 1$ & $60.40(12)$ & $\mathrm{C} 12-\mathrm{C} 11-\mathrm{C} 15-\mathrm{Fe} 1$ & $59.22(13)$ \\
\hline $\mathrm{C} 7-\mathrm{C} 8-\mathrm{C} 9-\mathrm{C} 10$ & $-0.5(2)$ & & \\
\hline
\end{tabular}

Symmetry code: (i) $-x+1,-y+1,-z+1$.

Hydrogen-bond geometry $\left(A,{ }^{\circ}\right)$

$\mathrm{Cg} 1, \mathrm{Cg} 2$ and $\mathrm{Cg} 3$ are the centroids of the $\mathrm{C} 6-\mathrm{C} 10, \mathrm{C} 11-\mathrm{C} 15$ and $\mathrm{C} 2 / \mathrm{O} 1 / \mathrm{C} 3-\mathrm{C} 5$ rings, respectively.

\begin{tabular}{lllll}
\hline$D-\mathrm{H} \cdots A$ & $D-\mathrm{H}$ & $\mathrm{H} \cdots A$ & $D \cdots A$ & $D-\mathrm{H} \cdots A$ \\
\hline $\mathrm{C} 3-\mathrm{H} 3 \cdots C g 1^{\mathrm{ii}}$ & 0.95 & 2.81 & $3.686(3)$ & 153 \\
$\mathrm{C} 8-\mathrm{H} 8 \cdots C g 2^{\mathrm{iii}}$ & 0.95 & 2.85 & $3.764(2)$ & 161 \\
$\mathrm{C} 10-\mathrm{H} 10 \cdots C g 3^{\mathrm{i}}$ & 0.95 & 2.68 & $3.2097(18)$ & 116 \\
\hline
\end{tabular}

Symmetry codes: (i) $-x+1,-y+1,-z+1$; (ii) $x, y, z+1$; (iii) $x+1 / 2,-y+3 / 2, z-1 / 2$. 